\title{
Evolution of Electronic Energy Levels in Krypton Clusters from the Atom to the Solid
}

\author{
J. Stapelfeldt, J. Wörmer, and T. Möller \\ II. Institut für Experimentalphysik, Universität Hamburg, Luruper Chaussee 149, \\ D-2000 Hamburg 50, Federal Republic of Germany \\ (Received 19 September 1988)
}

\begin{abstract}
We deduce the evolution of electronic excitations in $\mathrm{Kr}$ clusters $(N=2-3000)$ from fluorescence excitation spectroscopy with synchrotron radiation. In small clusters $(N \leq 30)$, a broad absorption band appears, slightly red shifted compared with the atomic $5 s(3 / 2)_{1}$ resonance line. Intermediate-size clusters $(50<N \leq 200)$ show absorption profiles which are mainly correlated with the surface excitons of the solid. The bulk excitons are pronounced only in large clusters $(N \geq 200)$. Absorption processes at the surface and inside the cluster correlate well to the surface-to-volume ratio of the clusters.

PACS numbers: $71.90 .+\mathrm{q}, 33.20 .-\mathrm{t}, 36.40 .+\mathrm{d}, 78.40 .-\mathrm{q}$
\end{abstract}

Following Hoare, ${ }^{1}$ a cluster or microcluster may be defined as an aggregate, whether of atoms, molecules, and so on, so small that an appreciable proportion of these units must be present in its surface. Remarkable progress in the area of supersonic jets and cluster beams ${ }^{2,3}$ provides the basis for the exciting field of cluster physics. In the past decade, a large number of very interesting features such as "fivefold symmetries," 4 "magic numbers," 5,6 or size-dependent fragmentation processes ${ }^{7}$ have been observed. All these findings clearly demonstrate that clusters not only bridge the gap between molecules and solids ${ }^{8}$ but also have very specific properties.

As mentioned above, one unique aspect of cluster physics is the large surface-to-volume ratio. However, the discrimination between atoms at the surface and inside the cluster is only appropriate for clusters with $N>13$ atoms/cluster (for rare-gas clusters). In macroscopic crystals the ratio of surface-to-volume atoms is on the order of $10^{-5}-10^{-7}$; nevertheless, surface excitations were observed in thin films. ${ }^{9}$ For studies probing the large surface-to-volume ratio of clusters, an extended range of cluster size has to be taken into account because this ratio shows only a small variation with $N^{-1 / 3}$. $^{1}$

In this Letter we report on the first investigation of the evolution of electronic energy levels from the dimer to microcrystals of about 3000 atoms/particle. The measurements were performed on krypton clusters by use of fluorescence excitation spectroscopy with synchrotron radiation. ${ }^{10}$ The results allow a discrimination between surface and bulk atoms by spectroscopic means. The absorption of "surface" and "bulk" material is related to the cluster size in a very simple way, provided the clusters contain more than about 50 atoms.

The measurements were carried out at the new cluster-beam experiment CLULU, ${ }^{11}$ behind the SUPERLUMI station at HASYLAB, Hamburg. ${ }^{12}$ The clusters are generated in an adiabatic expansion through a small nozzle. Spectrally selected synchrotron radiation with a band pass of $0.05-0.25 \mathrm{~nm}$ was used to excite the cluster beam in the wavelength range from 110 to 150 $\mathrm{nm}$. The cluster-beam setup consists of two vacuum chambers. The first one contains an interchangeable, temperature-controlled nozzle $(T \geq 80 \mathrm{~K})$. It is pumped down to $10^{-1}$ mbar . . 1 $10^{-5}$ mbar with a base pump. The cluster beam enters the main chamber through a skimmer and then crosses the focused photon beam. At a band pass of $0.25 \mathrm{~nm}$, the photon flux in the intersection region is about $10^{11}-10^{12}$ photons/s. The main chamber is pumped down to a pressure between $5 \times 10^{-4}$ and $10^{-8}$ mbar. The measurements were performed with a conical nozzle (inner diameter $80 \mu \mathrm{m}$, cone angle $2 \Theta=25^{\circ}$, length $15 \mathrm{~mm}$ ) or with simple orifices of 80 and $800-\mu \mathrm{m}$ diameter. Relatively large skimmers of 2.5 or $5 \mathrm{~mm}$ in diameter were used to achieve an optimal overlap with the light beam. The stagnation pressure ranged between 0.1 and 5 bar at temperatures between 125 and $300 \mathrm{~K}$. Kr gas with a purity of $99.99 \%$ was used. The fluorescence light was collected perpendicular to both beams with a large spherical mirror and focused on the CsTe photocathode of a channel plate detector with a $\mathrm{MgF}_{2}$ window. The counting rates ranged between a few hundred and $\cong 10^{5} \mathrm{cps}$.

It must be pointed out that the determination of the size of neutral rare-gas clusters is a severe problem, especially in the range between $N=5-50$ atoms/cluster. Farges et al. ${ }^{13}$ have determined the size of neutral argon clusters in the range $N=50-750$ using electron diffraction measurements. Considering that the cluster size only depends on the characteristic properties of the gas, the stagnation pressure $p_{0}$, source temperature $T_{0}$, and the nozzle geometry ("principle of corresponding beams"), we can determine the cluster size in our beam making use of Hagena's scaling laws. ${ }^{14}$ For a given nozzle diameter, the mean cluster size $N$ is proportional to $P_{0}^{1.95}$ (simple orifice) or $P_{0}^{1.3}$ (conical nozzle). ${ }^{3,13}$ For small clusters $(N \leq 5)$ we took the data of Buck and Meyer ${ }^{15,16}$ for the calibration. In the region in between $(5<N<50)$, we can give only rough estimates based on extrapolations of the data of Buck and Meyer ${ }^{15,16}$ and 
Farges et al. ${ }^{13}$ We do not have to worry about fragmentation because the data used for the calibration are obtained for neutral clusters. Systematic deviations from the true cluster size should be smaller than a factor of 2 or 3. The width of the size distribution corresponds nearly to the mean cluster size $N .{ }^{13}$

Figure 1 shows fluorescence excitation spectra of $\mathrm{Kr}$ clusters as a function of cluster size in the vicinity of the
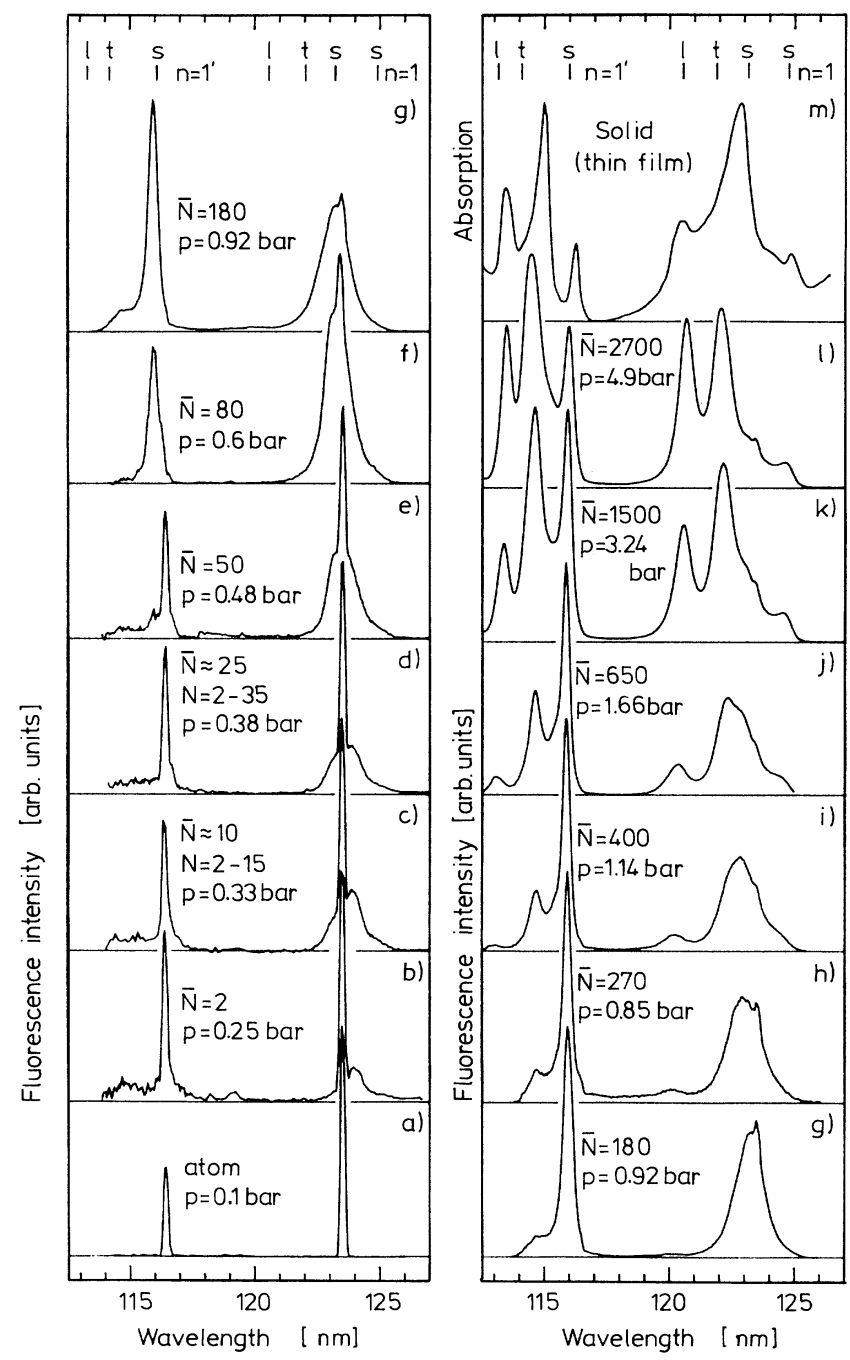

FIG. 1. Fluorescence excitation spectra $\left(\lambda_{\mathrm{fl}}=112-250 \mathrm{~nm}\right)$ of a krypton cluster beam as a function of mean cluster size $N$ in comparison with an absorption spectrum $(\mathrm{m})$ of a thin film $(30 \AA)$ of solid $\mathrm{Kr}$ recorded under oblique $\left(73^{\circ}\right)$ incidence (Ref. 17). The measurements on atoms, dimers, and small clusters [(a)- $(\mathrm{g})]$ are obtained with an orifice of $80 \mu \mathrm{m}$ at 144 $\mathrm{K}$, for the larger clusters [(h)-(k)] a conical nozzle $(d=80$ $\left.\mu \mathrm{m}, 2 \Theta=25^{\circ}\right)$.was used at room temperature. Energetic positions of surfaces $(s)$, longitudinal $(l)$, and transverse $(t)$ exciton contributions ( $n=1$ and $n=1^{\prime}$ excitons) are indicated in (g) and (m) (Ref. 18). atomic resonance lines $5 s(3 / 2)_{1}$ and $5 s^{\prime}(1 / 2)_{1}$. For comparison purposes, an absorption spectrum of a thin film of solid $\mathrm{Kr}$ (angle of incidence $73^{\circ}$ ) was added [Fig. $1(\mathrm{~m})]^{17}$ Except for the atomic resonance line, the cluster beam is optically thin. From solid-state ${ }^{19}$ and gascell $^{20}$ fluorescence experiments, it is known that the quantum efficiency is approximately constant in the range of excitation covered in Fig. 1. Provided this is also true for clusters, the fluorescence excitation spectra then display the absorption coefficient in relative units. At low stagnation pressures the beam consists mainly of atoms. The mean cluster size $N$ indicated in the figure concerns only the condensed part of the beam because cluster excitations and atomic excitations (resonance lines) are clearly separated. At the lowest stagnation pressure [Fig. 1(a)] only the atomic resonance lines are present. The width is mainly caused by the band pass of excitation. All absorption features of clusters seem to be much broader, not to mention that a fine structure might exist below our band pass of excitation.

For the discussion of the different absorption features observed and their variation with cluster size, it is useful to start with large clusters and end up with the dimer. For the largest clusters $[N=2700$, Fig. 1(1)] nearly a 1:1 correspondence to the properties of a thin film of solid $\mathrm{Kr}$ is found [Fig. $1(\mathrm{~m})$ ], neglecting small deviations of the energetic positions. The features at $124.7,123.1$, and $116 \mathrm{~nm}$ correspond to the well-known surface excitons of the solid, 9,21 whereas the pronounced maxima at 122.1 and $120.6 \mathrm{~nm}$ correspond to the transverse and longitudinal $n=1$ exciton, the maxima at 114.5 and $113.5 \mathrm{~nm}$ correspond to the transverse and longitudinal $n=1^{\prime}$ exciton. ${ }^{18}$ Since the transverse and longitudinal excitons are described as bandlike excitations, they are expected only in large clusters. Observation of longitudinal bulk excitons is usually restricted to electron energyloss spectroscopy. ${ }^{22}$ In optical $(k \cong 0)$ normal-incidence transmission experiments, the longitudinal excitons normally do not couple to the incident transverse electromagnetic field. However, in reflection geometry and for rough surfaces, optical excitation of longitudinal modes becomes possible. ${ }^{17}$ Considering that clusters have curved surfaces, this might explain the occurrence of longitudinal excitons. Both longitudinal and transverse excitons are prototypes of bulk excitations. With decreasing cluster size they get weaker in comparison to the surface excitations. At $n=270$, they only contribute about $15 \%$ to the observed fluorescence intensity [Fig. $1(\mathrm{~h})]$.

The intensity ratio of bulk and surface excitations displays a remarkable variation with cluster size and will be discussed below. Intermediate-size clusters $(50<N$ $<200$ ) show absorption profiles [Figs. 1(e)-1(g)] which are mainly correlated with the surface excitations of the solid. Bands corresponding to the longitudinal $n=1$ and $n=1^{\prime}$ excitons are nearly absent, whereas the bands cor- 
responding to the transverse excitons contribute a small amount to the spectra. For small clusters with $N \cong 10-25$ atoms/cluster [Figs. 1(c) and 1(d)] we observe only a broad band in the vicinity of the first atomic line at $123.6 \mathrm{~nm}$. A corresponding band in the vicinity of the second resonance line at $116.5 \mathrm{~nm}$ might be hidden in the noise, because of the reduced sensitivity of our setup at shorter wavelengths. Approaching the dimer case [Fig. 1(b)], the broad band is slightly shifted to longer wavelengths. Of course, the broad structure is partly due to the broad cluster-size distribution, but even at an estimated cluster size $N=25-50$ atoms/cluster, a close similarity to the surface exciton at $123.1 \mathrm{~nm}$ is found. ${ }^{9,21}$ A comparison of our measurements on small clusters with reflectivity measurements in dense gas and the liquid ${ }^{23}$ shows, in general, similarities but also striking differences which will be discussed later on. ${ }^{24}$

Here we discuss the intensity ratio of the fluorescence of surface excitations $J_{s}$ to the total fluorescence $J$ in more detail. This ratio is determined for the bands associated with the second resonance line at $116.5 \mathrm{~nm}$. Here surface and bulk excitations are clearly spectrally separated. If we assume that bulk and surface excitations have the same oscillator strength and that atoms at the surface contribute only to surface excitations, whereas all atoms inside the cluster contribute to bulk excitations, then the intensity ratio $J_{s} / J$ should be related to the cluster size $N$ in a very simple way. The proportion of surface atoms of small cluster has been discussed in detail by Hoare. ${ }^{1}$ If a cluster is approximately spherical, the number of surface atoms $N_{s}$ is related to $N$ by

$$
\frac{N_{s}}{N}=\frac{4 \pi R^{2} / \pi r^{2}}{R^{3} / r^{3}}=4 N^{-1 / 3}, \text { or } \frac{N_{s}}{N} N^{1 / 3}=4
$$

where $r$ is the radius of the atom and $R$ is the radius of the cluster.

Figure 2 shows a plot of the product $J_{s} / J N^{1 / 3}$ vs $N$ and additionally some corresponding points of an improved version of relation (1) for closed-shell icosahedra. ${ }^{1}$ The points for $N \leq 200$ are derived from measurements taken with a $800-\mu \mathrm{m}$ nozzle and which are not displayed in Fig. 1. With the assumptions mentioned above, the product $J_{s} / J N^{1 / 3}$ should roughly give a constant value of 4 . Indeed, Fig. 2 shows this predicted behavior, apart from small deviations. In other words, the intensity ratio of surface to bulk excitations is nearly equal to the ratio of surface atoms to atoms inside the cluster. In large clusters $(N \geq 1000)$ we observe systematic lower values because then the cluster diameter exceeds the penetration depth of the photons. Extrapolating our results to very small clusters, one could, in principle, expect that the first closed-shell icosahedron with $N=13$ should display surface as well as bulk excitations. Whether this description is appropriate for this cluster size is an open question. In our experiment we

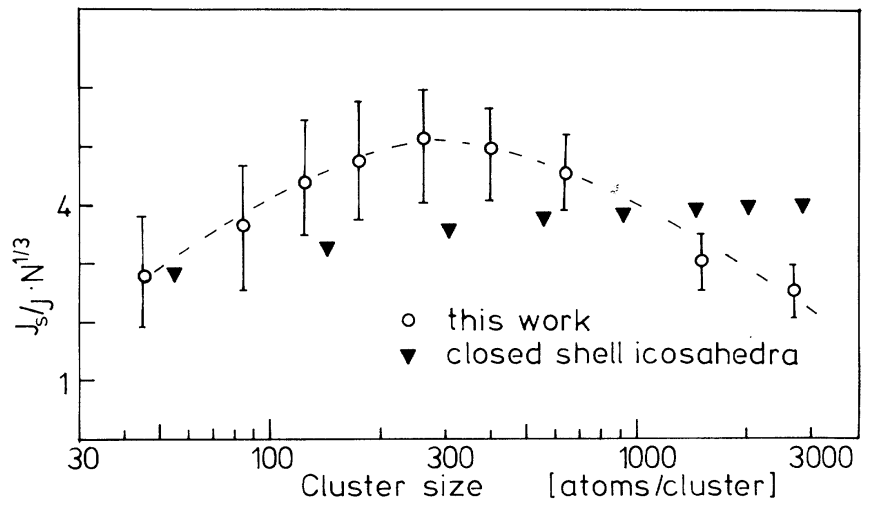

FIG. 2. Ratio of fluorescence intensity $J_{s}$ of surface excitations to total fluorescence intensity $J$ times $N^{1 / 3}$ vs cluster size $N$ derived for the excitations in the vicinity of the second atomic resonance line at $115.6 \mathrm{~nm}$. Some corresponding points for closed-shell icosahedra are included for comparison (Ref. 1).

did at least observe bulk excitations at $\cong 115 \mathrm{~nm}$ in beams with $N \cong 50$ atoms/cluster.

As a conclusion, we would like to point out that the bulk properties of $n=1$ and $n=1^{\prime}$ excitations appear in rather small clusters of about 50-100 atoms/cluster and are well pronounced in large clusters $(N \geq 200)$. A better determination of the lower limit is directly connected to the production of mass-selected cluster beams or mass-selective spectroscopic techniques. The energetic positions of characteristic absorption bands of krypton clusters are only slightly shifted compared to the corresponding exciton bands in the solid, while the relative intensities show a remarkable variation with the cluster size. This behavior may be explained by the classification of nearly all observed features as surface or bulk excitations. Within certain limits $(50<N<3000)$ the intensity ratio of surface to bulk excitations is nearly equal to the ratio of surface atoms to atoms inside the cluster. In other words, the large surface-to-volume ratio of clusters is directly observed by spectroscopic means.

The authors are grateful to Professor G. Zimmerer for strong support and fruitful discussions. We would also like to thank Professor U. Buck for the cluster-size calibration of small clusters and Dr. P. Gürtler, Dr. R. Reininger, and Dr. V. Saile for helpful discussions. Finally, we would like to thank W. Fechte for technical assistance. This work is supported by the Bundesministerium für Forschung und Technology under Grant No. 05305 AX B7 C3-14.

\footnotetext{
${ }^{1}$ M. R. Hoare, Adv. Chem. Phys. 40, 49 (1979).

${ }^{2}$ E. W. Becker, K. Bier, and W. Henkes, Z. Phys. 146, 333 (1956).
} 
${ }^{3}$ O. F. Hagena and W. Obert, J. Chem. Phys. 56, 1793 (1972).

${ }^{4}$ J. Farges, M. F. de Ferandy, B. Raoult, and G. Torchet, J. Chem. Phys. 78, 5067 (1983).

${ }^{5}$ O. Echt, K. Sattler, and E. Recknagel, Phys. Rev. Lett. 47, 1121 (1981).

${ }^{6}$ W. D. Knight, K. Clemenger, W. A. de Heer, W. A. Saunders, M. Y. Chou, and M. L. Cohen, Phys. Rev. Lett. 52, 2141 (1984).

${ }^{7}$ U. Buck and H. Meyer, Phys. Rev. Lett. 52, 109 (1984).

${ }^{8}$ J. Jortner, D. Scharf, and U. Landmann, in Excited State Spectroscopy in Solids, edited by U. M. Grassano and N. Terzi (North-Holland, Amsterdam, 1987), pp. 438-480.

${ }^{9}$ V. Saile, M. Skibowski, W. Steinmann, P. Gürtler, E. E. Koch, and A. Kozevnikov, Phys. Rev. Lett. 37, 305 (1976).

${ }^{10}$ T. Möller and G. Zimmerer, Phys. Scr. T17, 177 (1987).

${ }^{11}$ J. Stapelfeldt, J. Wörmer, G. Zimmerer, and T. Möller, to be published.

${ }^{12}$ H. Wilcke, W. Böhmer, R. Haensel, and N. Schwentner, Nucl. Instrum. Methods Phys. Res. 208, 59 (1983).
${ }^{13}$ J. Farges, M. F. de Ferandy, B. Raoult, and G. Torchet, J. Chem. Phys. 84, 3491 (1986).

${ }^{14}$ O. F. Hagena, Z. Phys. D 4, 291 (1987).

${ }^{15}$ U. Buck and H. Meyer, Surf. Sci. 156, 275 (1985).

${ }^{16} \mathrm{U}$. Buck, private communication.

${ }^{17} \mathrm{~V}$. Saile, in Electronic Excitations in Condensed Rare Gases, edited by N. Schwentner, E. E. Koch, and J. Jortner, Springer Tracts in Modern Physics Vol. 107 (Springer-Verlag, Berlin, 1985), p. 44.

${ }^{18}$ V. Saile, Appl. Opt. 19, 4115 (1980).

${ }^{19}$ E. Roick, Ph.D. thesis, Universität Hamburg, 1984 (unpublished).

${ }^{20}$ R. Brodmann, G. Zimmerer, and U. Hahn, Chem. Phys. Lett. 41, 164 (1976).

${ }^{21}$ V. Saile, Ph.D thesis, Universität München, 1976 (unpublished).

${ }^{22}$ N. Schwentner, E. E. Koch, and J. Jortner, in Ref. 7, p. 45.

${ }^{23}$ P. Laporte, J. L. Subtil, R. Reininger, V. Saile, S. Bernstorff, and I. T. Steinberger, Phys. Rev. B 35, 6270 (1987).

${ }^{24}$ J. Wörmer, J. Stapelfeldt, and T. Möller, to be published. 